\title{
A double-blinded randomised controlled trial exploring the effect of anodal transcranial direct current stimulation and uni-lateral robot therapy for the impaired upper limb in sub-acute and chronic stroke
}

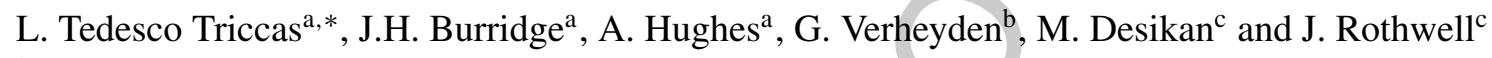

${ }^{\text {a }}$ Faculty of Health Sciences, University of Southampton, Southampton, UK

${ }^{\mathrm{b}}$ KU Leuven - University of Leuven, Department of Rehabilitation Sciences, Leuven, Belgium

${ }^{\mathrm{c}}$ Sobell Department of Motor Neuroscience, Institute of Neurology, University College of London, London, UK

\begin{abstract}
.
BACKGROUND: Neurorehabilitation technologies such as robot therapy (RT) and transcranial Direct Current Stimulation (tDCS) can promote upper limb (UL) motor recovery after stroke.

OBJECTIVE: To explore the effect of anodal tDCS with uni-lateral and three-dimensional RT for the impaired UL in people with sub-acute and chronic stroke.

METHODS: A pilot randomised controlled trial was conducted. Stroke participants had 18 one-hour sessions of RT (Armeo ${ }^{\circledR}$ Spring) over eight weeks during which they received 20 minutes of either real tDCS or sham tDCS during each session. The primary outcome measure was the Fugl-Meyer assessment (FMA) for UL impairments and secondary were: UL function, activities and stroke impact collected at baseline, post-intervention and three-month follow-up.

RESULTS: 22 participants (12 sub-acute and 10 chronic) completed the trial. No significant difference was found in FMA between the real and sham tDCS groups at post-intervention and follow-up $(p=0.123)$. A significant 'time' $\mathrm{x}$ 'stage of stroke' was found for FMA $(p=0.016)$. A higher percentage improvement was noted in UL function, activities and stroke impact in people with sub-acute compared to chronic stroke.
\end{abstract}

CONCLUSIONS: Adding tDCS did not result in an additional effect on UL impairment in stroke. RT may be of more benefit in the sub-acute than chronic phase.

Keywords: Transcranial direct current stimulation, robot therapy, stroke, rehabilitation, upper limb, motor recovery

\section{Background}

Approximately one third of stroke survivors experience disability; motor impairments are recognised as a major contributor (Dimyan \& Cohen, 2011). This

\footnotetext{
*Address for correspondence: Lisa Tedesco Triccas, Building 45, Faculty of Health Sciences, University of Southampton, SO17 1BJ Southampton, UK. Tel.: +44 (0) 1603 591686; E-mail: 1.tedescotriccas@uea.ac.uk.
}

disability is often long-term and is one of the primary reasons for psychosocial impact experienced by people with stroke, their families and the healthcare system (Aprile et al., 2008).

As part of stroke care, rehabilitation techniques are thought to contribute to recovery by promoting anatomical and physiological re-organisation of damaged networks (Dobkin \& Dorsch, 2013) which may be remodelled or unmasked by motor practice (Nudo, 
Wise, SiFuentes, \& Milliken, 1996; Toni, Krams, Turner, \& Passingham, 1998; Ziemann, Muellbacher, Hallett, \& Cohen, 2001). This re-organisation is then believed to promote UL motor recovery. However, at six months post-stroke, $33 \%$ to $66 \%$ do not present with recovery of UL function and only a small percentage of 5-20\% achieve full recovery (Kwakkel \& Kollen, 2013; Kwakkel, Kollen, van der Grond, \& Prevo, 2003).

Interventions such as Robot Therapy (RT) are currently being investigated as part of UL rehabilitation research programmes for people after stroke (Tanaka, Sandrini, \& Cohen, 2011). Robotic devices can provide repetitive, high-intensity, task-specific, interactive, cost-effective intervention of the impaired UL, can measure changes whilst providing feedback to people with stroke and can potentially be more effective than conventional therapy (Hesse, Werner, Kabbert, \& Buschfort, 2014; Klamroth-Marganska et al., 2014; Prange, Jannink, Groothuis-Oudshoorn, Hermens, \& IJzerman, 2006; Wagner et al., 2011). RT has been shown to result in improvement of motor function of the UL; however, it may not significantly improve strength and activities of daily living and dexterity (Basteris et al., 2014; Mehrholz, Haedrich, Platz, Kugler, \& Pohl, 2012). Thus, attention has focussed on trying to improve outcome of therapy by combining RT with other approaches such as transcranial direct current stimulation (tDCS).

Nitsche and Paulus (2000) demonstrated that weak, direct currents, applied transcranially, cause polaritydependent changes in excitability of human motor cortex (Brunoni et al., 2011). tDCS, a non-invasive method of brain stimulation applies a DC current of 1-2 $\mathrm{mA}$ via saline-soaked sponge electrodes and is thought to modulate neural excitability in brain areas by polarising the membranes of cortical neurones and modulating their firing patterns. In addition to its immediate effects on neural discharge, $\mathrm{tDCS}$ appears to cause after-effects on cortical excitability. These depend on the strength, duration and polarity of the stimulation and can outlast the period of stimulation by up to one hour (Nitsche et al., 2005) (Nitsche and Paulus, 2001, Nitsche et al., 2003b). The longer lasting effects have been attributed to synaptic changes which involve modification of the synaptic strength of N-methyl-Daspartate receptors or altering Gamma-Aminobutyric Acid (GABA) activation in the remaining motor areas of the cortex (Liebetanz, Nitsche, Tergau, \& Paulus, 2002; Stagg et al., 2009). Because of this, tDCS is sometimes said to promote long term potentiation and neuroplasticity (Fritsch et al., 2010).
It has been postulated that combining tDCS with rehabilitation can increase neuroplasticity and improve the response of UL motor impairments and function to rehabilitation. Several small studies in people with sub-acute and chronic stroke have found that there is a significant improvement in UL motor impairments and activities when real tDCS has been coupled with conventional therapy or virtual reality as compared to sham stimulation (Khedr et al., 2013; Kim et al., 2010; Lee \& Chun, 2014; Nair, Renga, Lindenberg, Zhu, \& Schlaug, 2011; Viana et al., 2014; Wu et al., 2013).

The present study was designed to assess whether the effectiveness of RT in improving UL impairments in sub-acute and chronic stroke could be enhanced by concurrent tDCS. As in previous studies, we used anodal tDCS over the affected hemisphere since it produces excitatory effects on corticospinal excitability in healthy individuals. Anodal tDCS has been reported to improve UL reaction times in people with chronic stroke (Hummel et al., 2005; Charlotte Jane Stagg et al., 2011); it has been linked with decrease in GABA levels of the underlying motor cortex; and it is reported to increase functional connectivity within the motor areas of the brain (Stagg et al., 2009). There is only one previous study which combined anodal tDCS and RT in 96 people with sub-acute stroke. It did not result in significant UL motor improvements (Hesse et al., 2011). This could have been due to the choice of RT which only focused on distal and bilateral UL movements. Our protocol attempted to address this deficiency by adding anodal tDCS to three-dimensional and uni-lateral RT for sub-acute/chronic stroke survivors with UL impairment. A total of 22 patients underwent 18 sessions of RT or RT combined with tDCS over an eight-week period (approximately two/three sessions per week) and were followed up 3 months later. The primary aim of the study was to examine the effectiveness of additional tDCS; in addition we conducted an exploratory analysis to test whether time after stroke affected the response to therapy.

\section{Methods}

The research design was a double-blinded pilot RCT. Registered protocol number: NCT01405378.

\subsection{Criteria and Recruitment}

Participants included were 18 years and above; had a confirmed clinical diagnosis of stroke by a neurologist or stroke specialist, no previous history of another 
stroke, were $>2$ weeks post-stroke, had upper and forearm and hand paresis (Medical Research Council scale for muscle strength $>2$ ) with minimal spasticity allowed (Modified Ashworth scale $\leq 2$ ) and partial shoulder flexion with gravity. They also had to have good sitting balance and ability to provide informed consent. Participants were excluded if they had: impaired gross cognitive function ( $<24$ on the Mini-Mental State Examination (Folstein, Folstein, \& McHugh, 1975), any another neurological condition apart from stroke, shoulder pain resulting from shoulder flexion beyond $90^{\circ}$, epilepsy, implants in the brain, previous brain neurosurgery, metal implants in the skull or brain including cochlear implants, medications that influence cortical excitability, previous adverse effects when stimulated with tDCS and pregnancy.

Participants were recruited from seven United Kingdom National Health Service sites and a private neurology hospital. Potential participants were provided an information pack by their health care professional or research nurse on leaving the rehabilitation unit/hospital ward. Participants already in the community were informed about the trial at their home, at stroke groups or at the day hospital during their rehabilitation session.

\subsection{Protocol}

\subsubsection{Assessment and outcome measures}

Participants meeting the criteria and consenting to participate in the study were assessed at baseline, postintervention and three month follow-up, conducted in the laboratory of the University of Southampton. Three blinded assessors, trained qualified physiotherapists with experience in stroke assessment and neurological rehabilitation carried out clinical assessments (same assessor per participant for baseline, post-intervention and follow-up). The primary outcome measure of this study was the Fugl-Meyer Assessment (FMA) which is a quantitative and standardised clinical measure assessing motor recovery of the impaired upper limb in people with stroke (Fugl-Meyer, Jääskö, Leyman, Olsson, \& Steglind, 1975; Platz et al., 2005).

The secondary outcome measures were: (1) Action Research Arm Test (ARAT), an UL functional measure which involves testing the ability to grasp, move, and release objects differing in size, weight, and shape by the affected UL (Lyle, 1981), (2) Motor Activity Log28 (MAL), a semi-structured interview during which respondents rate how well (quality of movement scale) they use the paretic hand during 28 UL activities of daily living (Uswatte, Taub, Morris, Light, \& Thompson, 2006) and (3) Stroke Impact Scale (3.0) (SIS) which evaluates function and quality of life in eight clinically relevant domains on the basis of self-report (Duncan, Bode, Min Lai, \& Perera, 2003).

Before each RT intervention session, participants also carried out an additional two assessments on the Armeo ${ }^{\circledR}$ Spring robot (Hocoma AG, Switzerland); 'vertical catching' and 'A-Goal' which involved a target appearing on the screen and by moving the robotic arm the participants were able to reach that target by a cursor. Through these assessments the Hand Path Ratio (HPR) was measured which was calculated by the recorded distance between the cursor and the target divided by the straight line distance.

\subsubsection{Randomisation}

Block randomisation was used with a computer program called 'random allocation software' (Saghaei, 2004). Each participant was first stratified into a subacute (2-16 weeks post-stroke) or 'chronic' ( $>16$ weeks post-stroke) groups (chronic stage eliminating 'spontaneous recovery (Langhorne, Bernhardt, \& Kwakkel, 2011). Each participant was then randomised into group A or B; Group A: Anodal tDCS and RT, and Group B: Sham tDCS and RT.

To conceal allocation, an independent person placed the printed papers of sham/real in sealed opaque envelopes according to block randomisation. As soon as a participant enrolled in the study, the researcher made a telephone call to the independent person who then stated whether 'real' or 'sham' was to be administered to the participant.

\subsubsection{Intervention}

Each intervention session took approximately an hour and 15 minutes in total and was carried out at either at the Faculty of Health Sciences, University of Southampton or at Christchurch Day Hospital, Christchurch, depending on participant preference. The intervention programme comprised of 18 sessions during an eight-week period (approximately two/three sessions per week).

Real tDCS/sham tDCS was applied using the HDCkit ${ }^{\circledR}$ (Newronika, 2012) for the first 20 minutes of the one hour RT intervention session. The arbitrary C3 and C4 positions of the 10-20 EEG system (Klem, Lüders, Jasper, \& Elger, 1999) were measured for the placement of the anodal electrode over the affected M1 as carried out in previous tDCS studies (Hesse et al., 2011; Vines, Cerruti, \& Schlaug, 2008). Direct current 


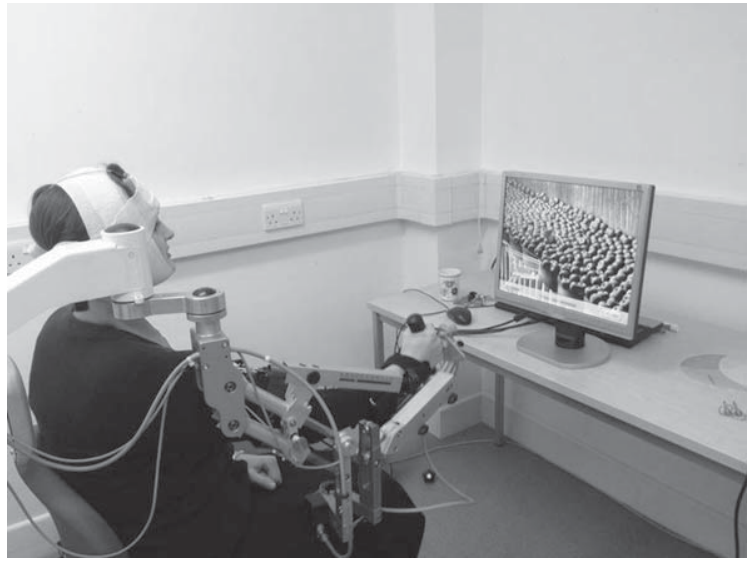

Fig. 1. A lady playing a computer generated fruit shopping game using the Armeo ${ }^{\circledR}$ Spring Robot. In the game, the person needed to manoeuvre the robot to pick up an apple and place it in the shopping cart as demonstrated on the computer screen.

was transferred by $35 \mathrm{~cm}^{2}(7 \times 5 \mathrm{~cm})$ rubber electrodes surrounded by saline-soaked pair of surface sponge electrodes using adhesive bandages. The anode was applied over the M1 area of the affected hemisphere and the cathode was positioned on the contralateral supraorbital region (Fig. 1). For the sham stimulation current faded-in and faded-out over 10 seconds at the beginning and end of the 20 minutes in a ramp-like fashion. Stimulation amplitude was $1 \mathrm{~mA}$.

The Armeo ${ }^{\circledR}$ Spring arm robot provided the robotic intervention, supporting the arm against gravity through integrated springs (Fig. 1). The robot provided a large three Dimensional workspace. The robotic arm has integrated sensors that measures kinematics, allowing participants to interact with therapeutic computer games and receive feedback about performance (Housman, Scott, \& Reinkensmeyer, 2009). The intervention targeted integrated movements involving the shoulder, elbow, wrist and grip of the impaired UL. The games and the rest intervals were determined by clinical need and after every session, the level of support was minimally decreased in order to encourage maximal effort by the participant.

\subsection{Data Analysis}

In addition to the clinical assessor, video recorded FMA and ARAT assessments were also scored by an additional blinded clinical assessor. The scores of each rater were matched and any disagreement was resolved by discussion between both assessors. Percentages of change from maximum score of each outcome measure post-intervention and follow-up were calculated for the sub-acute and chronic groups. Two-way ANOVA were applied to FMA, HPR, MAL and SIS data to test level of significance between the real and sham group using IBM SPSS Statistics Version 21 and Kruskal-Wallis tests was applied to the ARAT data. As an exploratory analysis, two-way repeated measures ANOVA were applied to all FMA, HPR, MAL and SIS data (both groups) +/-TDCS with 'time' point (baseline, postintervention and follow-up for all data but just baseline and post-intervention for HPR data) as within-subject factor and 'stage of stroke' (sub-acute or chronic) as in-between subject factor. Friedman ANOVA was applied to the ARAT data. The Greenhouse-Geisser correction was applied to correct for non-sphericity. The Paired-Samples $t$-test was used for post-hoc analysis of FMA, HPR, MAL and SIS data to compare means values between two time-points (i.e. baseline and post-intervention or baseline and follow-up scores) and Wilcoxon-Signed Rank test was applied to the ARAT data. Significant values were accounted at $p=\leq 0.05$. Power calculations were calculated based on the standard deviation found from the FMA baseline score of the sub-acute and chronic groups using a $t$-test comparing two independent samples (power of $80 \%$ and $p$ value of 0.05 ).

\section{Results}

Between March 2012-July 2013, from all the recruitment sites, 35 participants agreed to take part of which 23 participants were eligible. 12 of these were randomised to the real tDCS group and 11 to the sham group (Fig. 2). Details of the patients are given in Table 1 . There were no differences in age or UL impairments between the real and sham groups $(p=0.686$ and 0.55 respectively) and the mean FMA baseline score for the whole group was 32.3 (SD16.6).

After four intervention sessions, a participant with chronic stroke dropped out of the trial due to a skin reaction after receiving four real tDCS sessions, therefore 22 participants completed the trial. Each participant continued with their standard rehabilitation sessions of physiotherapy and occupational therapy (average twice a week) during the trial and follow-up period. Participants receiving real tDCS reported sensations of itching (58\%), tingling (58\%), warmth (58\%), burning (50\%), pain $(42 \%)$, light flashes $(33 \%)$ and headaches $(8 \%)$. After RT, participants reported fatigue (55\%), shoulder pain of affected side (32\%) and upper trapezius pain of affected side (14\%). 


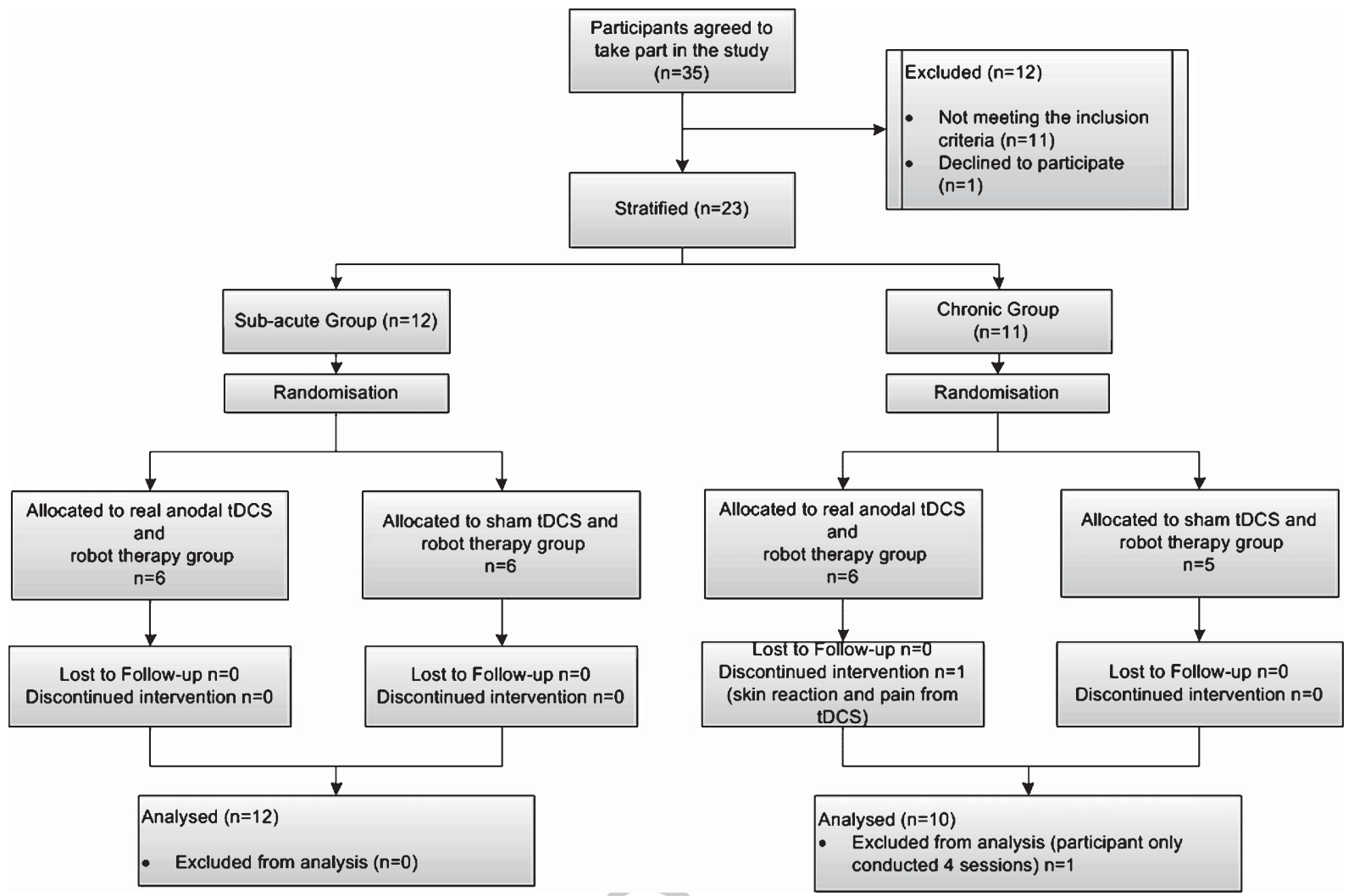

Fig. 2. Consort flow diagram: Process of participant recruitment to completion of study.

\subsection{Real tDCS Versus Sham tDCS and RT}

A two way ANOVA with group (real and sham tDCS) and time (FMA baseline, post-treatment and follow-up) showed a main effect of time $(F=24.157, \mathrm{df}=1.406$, $p=<0.001)$ but no effect of group or a group $\mathrm{X}$ time interaction $(F=3.070, \mathrm{df}=1, p=0.095)$. Thus the treatment improved FMA to the same extent whether patients received real or sham tDCS. Post-hoc analysis showed significant changes between post-intervention and baselines scores $(p=<0.001$ real and $p=<0.001$ sham; paired $t$-test) and follow-up and baseline scores ( $p=0.012$ real and $p=0.011$ sham; paired $t$-test) were found between FMA changes at three-month follow-up compared to post-intervention scores $(p=0.111$ sham and $p=0.071$ real; paired $t$-test).

A similar effect was observed for the HPR, ARAT, MAL and SIS. A two way ANOVA with group (real and sham tDCS) and time (baseline, posttreatment and follow-up) showed a main effect of time (HPR: $F=14.376, \mathrm{df}=1, p=0.002),(\mathrm{MAL}: F=8.537$, $\mathrm{df}=1.681, p=0.002), \quad($ SIS $: F=19.326, \mathrm{df}=1.605$, $p=<0.001$ ) but no effect of group or a group $\mathrm{X}$ time interaction (HPR: $F=1.015, \mathrm{df}=1, p=0.332$ ), (MAL: $F=0.228, \mathrm{df}=1, p=0.639$ ), (SIS: $F=0.477$, $\mathrm{df}=1, p=0.498)$. Friedman ANOVA/Kruskal-Wallis test with group (real and sham tDCS) and time (ARAT baseline, post-treatment and follow-up) showed a main effect of time $\left(X^{2}=16.636, \mathrm{df}=2, p=<0.001\right)$ but no effect of group or a group $\mathrm{x}$ time interaction $\left(X^{2}=1.403, \mathrm{df}=1, p=0.236 ; X^{2}=2.293, \mathrm{df}=1\right.$, $p=0.130$ respectively).

\subsection{Sub-Acute Versus Chronic Stage}

Each of the two treatment groups contained approximately equal numbers of acute and chronic stroke patients. Since there was no additional effect of tDCS, we combined the data from both groups and analysed whether treatment had a greater effect on sub-acute or chronic patients. There were no differences in age or UL impairments between the sub-acute and chronic groups ( $p=0.121$ and $p=0.249$ respectively). The mean baseline FMA score of the sub-acute group was 36.7 
Table 1

Demographic data of participants enrolled in the study

\begin{tabular}{|c|c|c|c|c|c|c|c|c|}
\hline $\begin{array}{l}\text { Participant } \\
\text { Number }\end{array}$ & $\begin{array}{l}\text { Gender } \\
{ }^{1}(\mathrm{M} / \mathrm{F})\end{array}$ & $\begin{array}{c}\text { Age } \\
\text { (years) }\end{array}$ & $\begin{array}{c}\text { Handed- } \\
\text { ness } \\
(\mathrm{L} / \mathrm{R})^{2}\end{array}$ & $\begin{array}{l}\text { Type of } \\
\text { Stroke } \\
(\mathrm{I} / \mathrm{H})^{3}\end{array}$ & $\begin{array}{c}\text { FMA } \\
\text { Baseline }\end{array}$ & $\begin{array}{c}\text { Time since } \\
\text { Stroke } \\
\text { (months) }\end{array}$ & $\begin{array}{l}\text { Sub-acute/ } \\
\text { Chronic } \\
\text { Stroke }\end{array}$ & $\begin{array}{l}\text { Location } \\
\text { of Stroke } \\
(\mathrm{SC} / \mathrm{C})^{4}\end{array}$ \\
\hline \multicolumn{9}{|l|}{ Sham Group } \\
\hline 1 & M & 52 & $\mathrm{R}$ & I & 36 & 2 & Sub-acute & R SC \\
\hline 2 & M & 71 & $\mathrm{R}$ & $\mathrm{H}$ & 52 & 3 & Sub-acute & R SC \\
\hline 3 & $\mathrm{~F}$ & 60 & $\mathrm{R}$ & I & 59 & 3 & Sub-acute & $\mathrm{L} \mathrm{SC}$ \\
\hline 4 & M & 78 & $\mathrm{R}$ & I & 46 & 2 & Sub-acute & $\mathrm{L} \mathrm{SC}$ \\
\hline 5 & $\mathrm{~F}$ & 83 & $\mathrm{R}$ & I & 49 & 2 & Sub-acute & $\mathrm{R} \mathrm{C}$ \\
\hline 6 & $\mathrm{~F}$ & 76 & $\mathrm{R}$ & I & 39 & 2 & Sub-acute & $\mathrm{LSC}$ \\
\hline 7 & M & 53 & $\mathrm{R}$ & I & 23 & 25 & Chronic & $\mathrm{R} \mathrm{C}$ \\
\hline 8 & M & 49 & $\mathrm{R}$ & I & 17 & 53 & Chronic & $\mathrm{R} \mathrm{C}$ \\
\hline 9 & M & 58 & $\mathrm{R}$ & $\mathrm{H}$ & 28 & 9 & Chronic & $\mathrm{LSC}$ \\
\hline 10 & M & 37 & $\mathrm{R}$ & I & 37 & 22 & Chronic & $\mathrm{L} \mathrm{C}$ \\
\hline 11 & $\mathrm{~F}$ & 71 & $\mathrm{R}$ & I & 22 & 24 & Chronic & $\mathrm{R} \mathrm{SC/C}$ \\
\hline \multicolumn{9}{|l|}{ Real Group } \\
\hline 12 & $\mathrm{~F}$ & 79 & $\mathrm{R}$ & I & 22 & 2 & Sub-acute & L (Unknown Location) \\
\hline 13 & M & 72 & $\mathrm{~L}$ & I & 4 & 3 & Sub-acute & R SC \\
\hline 14 & M & 68 & $\mathrm{R}$ & I & 40 & 2 & Sub-acute & $\mathrm{L} \mathrm{SC}$ \\
\hline 15 & $\mathrm{~F}$ & 47 & $\mathrm{R}$ & $\mathrm{H}$ & 59 & 3 & Sub-acute & $\mathrm{L} \mathrm{SC}$ \\
\hline 16 & $\mathrm{~F}$ & 57 & $\mathrm{R}$ & I & 8 & 3 & Sub-acute & R SC \\
\hline 17 & M & 63 & $\mathrm{R}$ & I & 26 & 2 & Sub-acute & $\mathrm{R} \mathrm{SC}$ \\
\hline 18 & M & 68 & $\mathrm{R}$ & I & 19 & 35 & Chronic & $\mathrm{R} \mathrm{C}$ \\
\hline 19 & $\mathrm{~F}$ & 48 & $\mathrm{R}$ & I & 23 & 21 & Chronic & $\mathrm{LC}$ \\
\hline 20 & M & 59 & $\mathrm{R}$ & $\mathrm{H}$ & 61 & 61 & Chronic & R SC \\
\hline 21 & M & 65 & $\mathrm{R}$ & $\mathrm{H}$ & 32 & 90 & Chronic & $\mathrm{L} \mathrm{SC}$ \\
\hline 22 & M & 71 & $\mathrm{R}$ & I & 8 & 72 & Chronic & $\mathrm{R} \mathrm{C}$ \\
\hline 23 & $\mathrm{~F}$ & 74 & $\mathrm{R}$ & I & 33 & 10 & Chronic & L SC \\
\hline \multirow[t]{2}{*}{$\%$ Mean $(\mathrm{SD})$} & $60.9 \% \mathrm{M}$ & $63.4(12.0)$ & $95.7 \% \mathrm{R}$ & $21.7 \% \mathrm{H}$ & 32.3 & 19.6 & $52.2 \%$ Sub-Acute & $31.8 \% \mathrm{C} 63.6 \% \mathrm{SC}$ \\
\hline & $39.1 \% \mathrm{~F}$ & & $4.3 \% \mathrm{~L}$ & $78.3 \% \mathrm{I}$ & (16.6) & $(25.7)$ & 47.8\% Chronic & $4.6 \% \mathrm{SC} / \mathrm{C}$ \\
\hline
\end{tabular}

${ }^{1} \mathrm{~F} / \mathrm{M}=$ Female/Male ${ }^{2} \mathrm{R} / \mathrm{L}=$ Right/Left ${ }^{3} \mathrm{I} / \mathrm{H}=$ Ischaemic/Haemorrhagic ${ }^{4} \mathrm{SC} / \mathrm{C}=$ Sub-Cortical/Cortical.

Table 2

Exploratory analysis of the mean FMA ${ }^{1}$ scores at baseline, post-intervention and follow-up of real and sham groups

\begin{tabular}{lccccccc}
\hline Mean $(\mathrm{SD})^{2}$ & Baseline & Post-intervention & Follow-up & Change $^{3}$ & $p$-value & Change $^{4}$ & $p$-value \\
\hline & $(\mathrm{B})$ & $(\mathrm{P})$ & $(\mathrm{F})$ & $(\mathrm{P}-\mathrm{B})(\%)$ & $(\mathrm{P}-\mathrm{B})$ & $(\mathrm{F}-\mathrm{B})(\%)$ & $(\mathrm{F}-\mathrm{B})$ \\
Real Group & $24.91(16.01)$ & $33.64(16.25)$ & $32.09(16.65)$ & $+8.73(13.23 \%)$ & $<0.001^{*}$ & $+7.18(10.88 \%)$ & $0.012^{*}$ \\
Sham Group & $37.09(13.57)$ & $44.82(16.29)$ & $44.18(18.08)$ & $+7.73(11.71 \%)$ & $<0.001^{*}$ & $+7.09(10.74 \%)$ & $0.011^{*}$ \\
\hline
\end{tabular}

${ }^{1} \mathrm{FMA}=$ Fugl-Meyer Assessment/ Maximum Score is $66,{ }^{2} \mathrm{SD}=$ Standard Deviation, ${ }^{3}$ Change $=\%$ from the Maximum Score, ${ }^{4}$ Paired-Samples $t$-test, $\left({ }^{*}\right)$ significant at $p=\leq 0.05$.

(SD 18.4) demonstrating overall moderate UL impairment (58\%); $25 \%$ were severely impaired, and $17 \%$ were mildly impaired (Lum, Burgar, Shor, Majmundar, \& Van der Loos, 2002). The mean FMA score of the chronic group was 27.55 (SD 13.77) with $46 \%$ severely impaired, $46 \%$ moderately impaired ad $9 \%$ mildly impaired (Table 2).

A two way ANOVA with stage (sub-acute or chronic) and time (FMA baseline and post-treatment and follow-up) revealed a significant stage $\mathrm{X}$ time interaction $(F=1.015, \mathrm{df}=1, p=0.016)$ indicating that the response to treatment differed in the two groups. The absolute change in scores was 10.3 (or $15.5 \%$ baseline) $(p=<0.001$; paired $t$-test) in FMA of the sub-acute group at post-intervention and 10.6 (16.0\% baseline) $(p=0.001$; paired $t$-test $)$ at follow-up. Whereas there was only a significant absolute change in scores of $5.8(8.8 \%$ baseline $)(p=0.001$; paired $t$-test $)$ in the chronic group at post-intervention but non-significant change of 3.0 ( $4.5 \%$ baseline) ( $p=0.092$; paired $t$-test) at follow-up (Table 3 ). No significant changes were found between FMA changes at three-month follow-up compared to post-intervention scores $(p=0.828$ subacute and $p=0.127$ chronic).

There was a significant absolute change in HPR, ARAT, MAL and SIS scores of 0.42 (25.0\% baseline) ( $p=0.008$; paired $t$-test), 15.00 (26.3\% baseline) ( $p=0.03$; Wilcoxon-Signed Rank test), 0.97 (19.4\% 
Table 3

Exploratory analysis of the mean FMA ${ }^{1}$ scores at baseline, post-intervention and follow-up of sub-acute and chronic groups

\begin{tabular}{lccccccc}
\hline Mean $(\mathrm{SD})^{* 2}$ & Baseline & Post-intervention & Follow-up & Change $^{* 3}$ & $p$-value*4 & Change & $p$-value \\
\hline & $(\mathrm{B})$ & $(\mathrm{P})$ & $(\mathrm{F})$ & $(\mathrm{P}-\mathrm{B})(\%)$ & $(\mathrm{P}-\mathrm{B})$ & $(\mathrm{F}-\mathrm{B})(\%)$ & $(\mathrm{F}-\mathrm{B})$ \\
Sub-Acute Group & $36.67(18.36)$ & $46.92(17.78)$ & $47.3(18.00)$ & $+10.25(15.53 \%)$ & $<0.001^{*}$ & $+10.58(16.03 \%)$ & $0.001^{*}$ \\
Chronic Group & $24.20(8.60)$ & $30.00(10.23)$ & $27.20(11.01)$ & $+5.80(8.78 \%)$ & $0.01^{*}$ & $+3.00(4.55 \%)$ & 0.092 \\
\hline
\end{tabular}

${ }^{* 1} \mathrm{FMA}=$ Fugl-Meyer Assessment/ Maximum Score is $66,{ }^{* 2} \mathrm{SD}=$ Standard Deviation, ${ }^{* 3}$ Change $=\%$ from the Maximum Score, ${ }^{* 4}$ Paired-Samples $t$-test, $\left({ }^{*}\right)$ significant at $p=\leq 0.05$.

baseline) ( $p=0.006$ paired $t$-test), $0.03(0.6 \%$ baseline) $(p=0.672)$ and $17.05(29.4 \%$ baseline $)(p=0.01$ paired $t$-test) respectively of the sub-acute group at post-intervention. Whereas there was a non-significant absolute change in HPR, ARAT, MAL and SIS scores of 0.17 ( $11.4 \%$ baseline) ( $p=0.09$; paired $t$-test), 2.00 (3.51\% baseline) $(p=0.176)$ and $0.39(0.7 \%$ baseline) $(p=0.168)$ respectively in the chronic group at post-intervention (Table 4). At follow-up, a significant absolute changes were found in ARAT, MAL and SIS scores of 16.50 (29.0\% baseline) ( $p=0.05$ WilcoxonSigned Rank Test), 1.25 (25.0\% baseline) ( $p=0.002$ paired $t$-test) and15.32 (26.4 baseline) $(p=<0.001$ paired $t$-test) for the sub-acute and only SIS was significant 3.54 (6.1\% baseline) $(p=0.005$ paired $t$-test $)$ in the chronic group (Table 4).

\section{Discussion}

This study explored the effect of combining anodal tDCS with uni-lateral and three dimensional RT for the impaired UL and stroke impact of participants with sub-acute and chronic stroke. The null hypothesis was accepted for the present study. Adding real tDCS to the RT programme did not result in significant differences in outcome compared to sham tDCS. After the intervention a larger significant reduction in UL impairments was observed in the sub-acute compared to the chronic participants.

A similar study explored the effects of RT and anodal, cathodal and sham tDCS in 96 participants with subacute stroke (Hesse et al., 2011). Even though the study by Hesse et al. involved a larger sample, non-significant differences were also reported on UL impairments. The latter study involved daily RT bi-lateral wrist RT daily sessions and applied an intensity of $2 \mathrm{~mA}$ as opposed to the $1 \mathrm{~mA}$ current used in the present study. A varied current intensity of $1 \mathrm{~mA}$ to $2 \mathrm{~mA}$ is also very prominent in similar recent studies involving tDCS and rehabilitation (Bolognini et al., 2011; Hesse et al., 2007; Khedr et al., 2013; Lindenberg, Renga, Zhu, Nair, \& Schlaug,
2010). Increasing the current of tDCS and involving daily RT sessions might not make a difference in the results. However, using RT as an intervention might be minimising the effects of anodal tDCS. Interventions such as standard UL rehabilitation programmes, virtual reality and constraint induced movement therapy in addition with tDCS have shown a significant improvement in UL impairments (Bolognini et al., 2011). This needs to be further explored with larger RCTs.

RT is a rehabilitation health technology which enforces intensive and repetitive task practice for the UL (Krakauer, 2006). In the present study, the percentage improvement of UL motor impairments from baseline to post-intervention was $16 \%$ in the sub-acute group as opposed to $9 \%$ in the chronic group. However, in both groups the absolute change in score was $>5$, meaning that there was a minimal clinically important difference was found for people in the sub-acute and chronic stage (Gladstone, Danells, \& Black, 2002; Page, Fulk, \& Boyne, 2012). Early improvement was expected in the participants in the sub-acute group due to spontaneous natural recovery involving surrounding areas to the lesion (restitution) (O'Dell, Lin, \& Harrison, 2009). Recovery also involves reorganisation of the brain tissue and learning and this occurs during the first six months post-stroke (Langhorne et al., 2011; O'Dell et al., 2009). For the chronic group, the intervention was the main factor that influenced the improvement since natural recovery could potentially be excluded. After several years post-stroke, UL impairment small improvements can still occur which might not be a result of true motor recovery but of compensatory movements (Levin, Kleim, \& Wolf, 2009). Therefore, integrating UL RT in community hospitals can potentially result in reduction of impairments which can last for three months and lower overall healthcare costs than traditional rehabilitation (Hesse, Werner, Kabbert, \& Buschfort, 2014; Wagner et al., 2011).

One of the main problems experienced by people with stroke is hand function. In order to grasp and release, the hand needs to open, position, grasp 


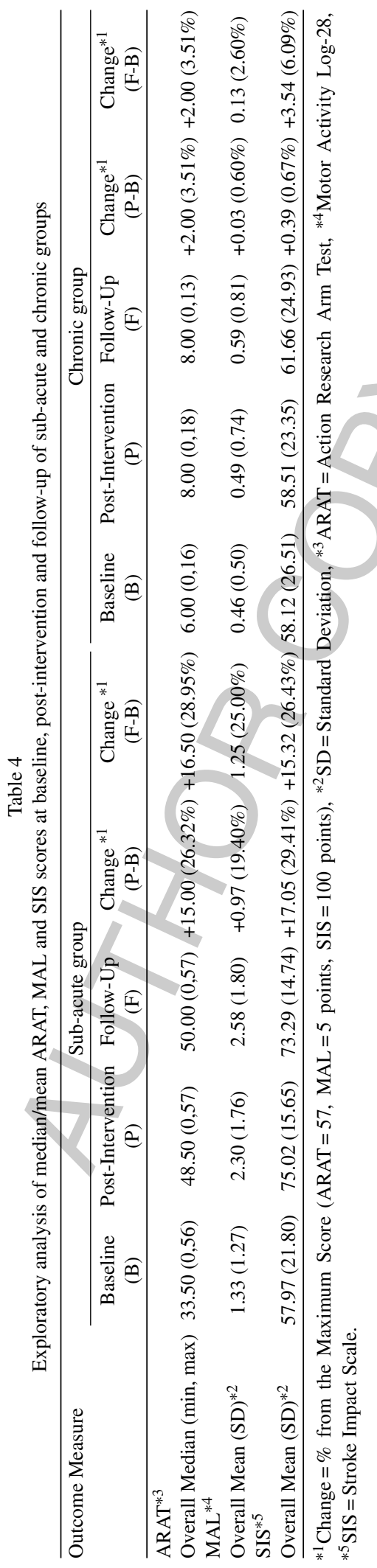


the object and then release that object (Connelly et al., 2010). It has been claimed that exoskeletons improve global UL movements but not hand dexterity (Lambercy et al., 2007). Participants with sub-acute stroke showed a greater improvement in UL function and dexterity than the chronic group. Constrained movement induced therapy, a rehabilitation technique that promotes hand use, has been combined with bihemispheric tDCS in 14 people with chronic stroke and significant differences in hand function were found in the real stimulation group compared to the sham stimulation group, which also remained stable at follow-up (Bolognini et al., 2011).

Participants in the sub-acute stage also showed a larger improvement in their stoke impact after the trial. Measuring stroke impact is important as part of the WHO's classification of disability (World Health Organisation, 2001). None of the previous studies involving tDCS have explored stroke impact and participation after the applied interventions. In stroke, prerequisites of participation usually involve the reintegration in the social community and perception of stroke recovery which can be measured by using the Stroke Impact Scale (Eriksson, Baum, Wolf, \& Connor, 2013). RT has been shown to significantly increase social participation compared to usual care (Lo et al., 2010).

A small and heterogeneous sample size was included in this study. The study would have been powered sufficiently to detect an effect of tDCS if it had been as large as reported in previous trials of non-robot therapy. Post-analysis a power calculation identified that 106 participants with sub-acute stroke and 94 participants with chronic stroke will be required to obtain a significant difference between real and sham tDCS groups. Additionally, participants were in a different phase (sub-acute and chronic), had different types, and locations of stroke, and a range of UL impairments. Also, participants continued concomitant treatment with an average of twice a week during the trial and the three-month follow-up period and therefore this could have had an impact in their UL recovery. The clinical assessments were carried out by three different assessors and therefore a potential increase in measurement error between the assessments could have occurred.

\section{Conclusion}

Conducting research is important to advance the knowledge on neurological rehabilitation of UL prob- lems that are commonly experienced by people with stroke. This research contributed to the current knowledge about combining tDCS to rehabilitation programmes in stroke. No significant differences were found between the real and sham tDCS groups. RT is potentially a more effective for people with sub-acute stroke, influencing UL impairment, function, activities and stroke impact. Larger studies need to be carried out in order for researchers and health care professionals to make more informed decisions when integrating tDCS and RT in stroke rehabilitation settings in the future.

\section{Acknowledgments}

We would like to thank the health care professionals, the participants and their carers for their support for this research. We would like to thank Dr Veronica Robles, Mrs Lindsay A. O'Connor, Mrs Claire Meagher and $\mathrm{Mr}$ Seng Kwee Wee for their contribution to data collection process for this trial. We also would like to thank Wessex Medical Research, University of Southampton and the Strategic Educational Pathways Scholarship Scheme (Malta) - The scholarship is partfinanced by the European Union - European Social Fund. Finally we would like to thank Dr Sean Ewings from the Southampton Statistical Sciences Research Institute at the University of Southampton, for his continual statistical support and advice.

\section{Declaration of interest}

None disclosed.

\section{References}

Aprile, I., Di Stasio, E., Romitelli, F., Lancellotti, S., Caliandro, P., Tonali, P., \& Padua, L. (2008). Effects of rehabilitation on quality of life in patients with chronic stroke. Brain Injury, 22(6), 451-456.

Basteris, A., Nijenhuis, S. M., Stienen, A. H., Buurke, J. H., Prange, G. B., \& Amirabdollahian, F. (2014). Training modalities in robotmediated upper limb rehabilitation in stroke: A framework for classification based on a systematic review. Journal of Neuroengineering and Rehabilitation, 11(1), 111.

Bolognini, N., Vallar, G., Casati, C., Latif, L. A., El-Nazer, R., Williams, J., \& Chessa, C. (2011). Neurophysiological and behavioral effects of tDCS combined with constraint-induced movement therapy in poststroke patients. Neurorehabilitation and Neural Repair, 25(9), 819-829.

Brunoni, A. R., Amadera, J., Berbel, B., Volz, M. S., Rizzerio, B. G., \& Fregni, F. (2011). A systematic review on reporting and assessment of adverse effects associated with transcranial 
direct current stimulation. Int J Neuropsychopharmacol, 14(8), 1133-1145.

Connelly, L., Jia, Y., Toro, M. L., Stoykov, M. E., Kenyon, R. V., \& Kamper, D. G. (2010). A pneumatic glove and immersive virtual reality environment for hand rehabilitative training after stroke. Neural Systems and Rehabilitation Engineering, IEEE Transactions on, 18(5), 551-559.

Dimyan, M. A., \& Cohen, L. G. (2011). Neuroplasticity in the context of motor rehabilitation after stroke. Nature Reviews Neurology, $7(2), 76-85$

Dobkin, B. H., \& Dorsch, A. (2013). New Evidence for Therapies in Stroke Rehabilitation. Current Atherosclerosis Reports, 15(6), 1-9.

Duncan, P. W., Bode, R. K., Min Lai, S., \& Perera, S. (2003). Rasch analysis of a new stroke-specific outcome scale: The stroke impact scale1. Archives of Physical Medicine and Rehabilitation, 84(7), 950-963.

Eriksson, G., Baum, M. C., Wolf, T. J., \& Connor, L. T. (2013). Perceived Participation After Stroke: The Influence of Activity Retention, Reintegration, and Perceived Recovery. The American Journal of Occupational Therapy, 67(6), e131-e138.

Folstein, M. F., Folstein, S. E., \& McHugh, P. R. (1975). MiniMental State: A practical method for grading the cognitive state of patients for the clinician: Journal of Psychiatric Research, 12, 189-198.

Fritsch, B., Reis, J., Martinowich, K., Schambra, H. M., Ji, Y., Cohen, L. G., \& Lu, B. (2010). Direct current stimulation promotes BDNF-dependent synaptic plasticity: Potential implications for motor learning. Neuron, 66(2), 198-204.

Fugl-Meyer, A., Jääskö, L., Leyman, I., Olsson, S., \& Steglind, S. (1975). The post-stroke hemiplegic patient. 1. a method for evaluation of physical performance. Scandinavian Journal of Rehabilitation Medicine, 7(1), 13.

Gladstone, D. J., Danells, C. J., \& Black, S. E. (2002). The FuglMeyer assessment of motor recovery after stroke: A critical review of its measurement properties. Neurorehabilitation and Neural Repair, 16(3), 232-240.

Hesse, S., Heß, A., Werner C, C., Kabbert, N., \& Buschfort, R. (2014). Effect on arm function and cost of robot-assisted group therapy in subacute patients with stroke and a moderately to severely affected arm: A randomized controlled trial. Clinical Rehabilitation, 28(7), 637-647.

Hesse, S., Waldner, A., Mehrholz, J., Tomelleri, C., Pohl, M., \& Werner, C. (2011). Combined Transcranial Direct Current Stimulation and Robot-Assisted Arm Training in Subacute Stroke Patients An Exploratory, Randomized Multicenter Trial. Neurorehabilitation and Neural Repair, 25(9), 838-846.

Hesse, S., Werner, C., Schonhardt, E., Bardeleben, A., Jenrich, W., \& Kirker, S. (2007). Combined transcranial direct current stimulation and robot-assisted arm training in subacute stroke patients: A pilot study. Restorative Neurology and Neuroscience, 25(1), 9-15.

Housman, S. J., Scott, K. M., \& Reinkensmeyer, D. J. (2009). A randomized controlled trial of gravity-supported, computerenhanced arm exercise for individuals with severe hemiparesis. Neurorehabilitation and Neural Repair, 23(5), 505-514.

Hummel, F., Celnik, P., Giraux, P., Floel, A., Wu, W.-H., Gerloff, C., \& Cohen, L. G. (2005). Effects of non-invasive cortical stimulation on skilled motor function in chronic stroke. Brain, 128(3), 490499.
Khedr, E. M., Shawky, O. A., El-Hammady, D. H., Rothwell, J. C., Darwish, E. S., Mostafa, O. M., \& Tohamy, A. M. (2013). Effect of Anodal Versus Cathodal Transcranial Direct Current Stimulation on Stroke Rehabilitation A Pilot Randomized Controlled Trial. Neurorehabilitation and Neural Repair, 27(7), 592-601.

Kim, D. Y., Lim, J. Y., Kang, E. K., You, D. S., Oh, M. K., Oh, B. M., \& Paik, N. J. (2010). Effect of transcranial direct current stimulation on motor recovery in patients with subacute stroke. American Journal of Physical Medicine \& Rehabilitation, 89(11), 879-886.

Klamroth-Marganska, V., Blanco, J., Campen, K., Curt, A., Dietz, V., Ettlin, T., \& Kollmar, A. (2014). Three-dimensional, task-specific robot therapy of the arm after stroke: A multicentre, parallel-group randomised trial. The Lancet Neurology, 13(2), 159-166.

Klem, G. H., Lüders, H., Jasper, H., \& Elger, C. (1999). The tentwenty electrode system of the International Federation. The International Federation of Clinical Neurophysiology. Electroencephalography and Clinical Neurophysiology. Supplement, 52, 3.

Krakauer, J. W. (2006). Motor learning: Its relevance to stroke recovery and neurorehabilitation. Current Opinion in Neurology, 19(1), 84-90.

Kwakkel, G., \& Kollen, B. (2013). Predicting activities after stroke: What is clinically relevant? International Journal of Stroke, 8(1), 25-32.

Kwakkel, G., Kollen, B. J., van der Grond, J., \& Prevo, A. J. H. (2003). Probability of Regaining Dexterity in the Flaccid Upper Limb: Impact of Severity of Paresis and Time Since Onset in Acute Stroke. Stroke, 34(9), 2181-2186.

Lambercy, O., Dovat, L., Johnson, V., Salman, B., Wong, S., Gassert, R., \& Burdet, E. (2007). Development of a robot-assisted rehabilitation therapy to train hand function for activities of daily living. Paper presented at the Rehabilitation Robotics, 2007. ICORR 2007. IEEE 10th International Conference.

Langhorne, P., Bernhardt, J., \& Kwakkel, G. (2011). Stroke rehabilitation. The Lancet, 377(9778), 1693-1702.

Lee, S. J., \& Chun, M. H. (2014). Combination transcranial direct current stimulation and virtual reality therapy for upper extremity training in patients with subacute stroke. Archives of Physical Medicine and Rehabilitation, 95(3), 431-438.

Levin, M. F., Kleim, J. A., \& Wolf, S. L. (2009). What do motor "recovery" and "compensation" mean in patients following stroke? Neurorehabilitation and Neural Repair, 23(4), 313-319.

Liebetanz, D., Nitsche, M. A., Tergau, F., \& Paulus, W. (2002). Pharmacological approach to the mechanisms of transcranial DC-stimulation-induced after-effects of human motor cortex excitability. Brain, 125(10), 2238-2247.

Lindenberg, R., Renga, V., Zhu, L., Nair, D., \& Schlaug, G. (2010). Bihemispheric brain stimulation facilitates motor recovery in chronic stroke patients. Neurology, 75(24), 2176-2184.

Lo, A. C., Guarino, P. D., Richards, L. G., Haselkorn, J. K., Wittenberg, G. F., Federman, D. G., \& Volpe, B. T. (2010). Robot-assisted therapy for long-term upper-limb impairment after stroke. New England Journal of Medicine, 362(19), 1772-1783.

Lum, P. S., Burgar, C. G., Shor, P. C., Majmundar, M., \& Van der Loos, M. (2002). Robot-assisted movement training compared with conventional therapy techniques for the rehabilitation of upper-limb motor function after stroke. Archives of Physical Medicine and Rehabilitation, 83(7), 952-959. 
Lyle, R. C. (1981). A performance test for assessment of upper limb function in physical rehabilitation treatment and research. International Journal of Rehabilitation Research, 4(4), 483-492.

Mehrholz, J., Haedrich, A., Platz, T., Kugler, J., \& Pohl, M. (2012). Electromechanical and robot-assisted arm training for improving generic activities of daily living, arm function, and arm muscle strength after stroke. Cochrane Database Syst Rev, CD006876.

Nair, D. G., Renga, V., Lindenberg, R., Zhu, L., \& Schlaug, G. (2011). Optimizing recovery potential through simultaneous occupational therapy and non-invasive brain-stimulation using tDCS. Restorative Neurology and Neuroscience, 29(6), 411-420.

Newronika. (2012). hdckit. Retrieved 16th Decemeber 2012, from http://www.newronika.it/?page_id=960\&lang=en

Nitsche, M., \& Paulus, W. (2000). Excitability changes induced in the human motor cortex by weak transcranial direct current stimulation. The Journal of Physiology, 527(3), 633-639.

Nitsche, M. A., Seeber, A., Frommann, K., Klein, C. C., Rochford, C., Nitsche, M. S., \& Antal, A. (2005). Modulating parameters of excitability during and after transcranial direct current stimulation of the human motor cortex. The Journal of Physiology, 568(1), 291-303.

Nudo, R. J., Wise, B. M., SiFuentes, F., \& Milliken, G. W. (1996). Neural Substrates for the Effects of Rehabilitative Training on Motor Recovery After Ischemic Infarct. Science, 272 (5269), 1791-1794.

O’Dell, M. W., Lin, C. C. D., \& Harrison, V. (2009). Stroke rehabilitation: Strategies to enhance motor recovery. Annual Review of Medicine, 60, 55-68.

Page, S. J., Fulk, G. D., \& Boyne, P. (2012). Clinically important differences for the Upper-extremity fugl-meyer scale in people with minimal to moderate impairment due to chronic stroke. Physical Therapy, 92(6), 791-798.

Platz, T., Pinkowski, C., van Wijck, F., Kim, I.-H., di Bella, P., \& Johnson, G. (2005). Reliability and validity of arm function assessment with standardized guidelines for the Fugl-Meyer Test, Action Research Arm Test and Box and Block Test: A multicentre study. Clinical Rehabilitation, 19(4), 404-411.

Prange, G. B., Jannink, M. J., Groothuis-Oudshoorn, C. G., Hermens, H. J., \& IJzerman, M. J. (2006). Systematic review of the effect of robot-aided therapy on recovery of the hemiparetic arm after stroke. Journal of Rehabilitation Research and Development, 43(2), 171.

Saghaei, M. (2004). Random allocation software for parallel group randomized trials. BMC Medical Research Methodology, 4(1), 26.
Stagg, C. J., Bachtiar, V., O'Shea, J., Allman, C., Bosnell, R. A., Kischka, U., \& Johansen-Berg, H. (2011). Cortical activation changes underlying stimulation-induced behavioural gains in chronic stroke. Brain, doi:10.1093/brain/awr313

Stagg, C. J., Best, J. G., Stephenson, M. C., O'Shea, J., Wylezinska, M., Kincses, Z. T., \& Johansen-Berg, H. (2009). Polarity-sensitive modulation of cortical neurotransmitters by transcranial stimulation. The Journal of Neuroscience, 29(16), 5202-5206.

Tanaka, S., Sandrini, M., \& Cohen, L. G. (2011). Modulation of motor learning and memory formation by non-invasive cortical stimulation of the primary motor cortex. Neuropsychological Rehabilitation, 21(5), 650-675.

Toni, I., Krams, M., Turner, R., \& Passingham, R. E. (1998). The time course of changes during motor sequence learning: A whole-brain fMRI study. Neuroimage, 8(1), 50-61.

Uswatte, G., Taub, E., Morris, D., Light, K., \& Thompson, P. (2006). The Motor Activity Log-28 Assessing daily use of the hemiparetic arm after stroke. Neurology, 67(7), 1189-1194.

Viana, R., Laurentino, G., Souza, R., Fonseca, J., Silva Filho, E., Dias, S., \& Monte-Silva, K. (2014). Effects of the addition of transcranial direct current stimulation to virtual reality therapy after stroke: A pilot randomized controlled trial. NeuroRehabilitation, 34, 437-446.

Vines, B. W., Cerruti, C., \& Schlaug, G. (2008). Dual-hemisphere tDCS facilitates greater improvements for healthy subjects' nondominant hand compared to uni-hemisphere stimulation. BMC Neuroscience, 9(1), 103.

Wagner, T. H., Lo, A. C., Peduzzi, P., Bravata, D. M., Huang, G. D., Krebs, H. I., \& Haselkorn, J. K. (2011). An economic analysis of robot-assisted therapy for long-term upper-limb impairment after stroke. Stroke, 42(9), 2630-2632.

Wagner, T. H., Lo, A. C., Peduzzi, P., Bravata, D. M., Huang, G. D., Krebs, H. I., \& Guarino, P. D. (2011). An Economic Analysis of Robot-Assisted Therapy for Long-Term Upper-Limb Impairment After Stroke. Stroke, 42(9), 2630-2632.

Wu, D., Qian, L., Zorowitz, R. D., Zhang, L., Qu, Y., \& Yuan, Y. (2013). Effects on decreasing upper-limb poststroke muscle tone using transcranial direct current stimulation: A randomized sham-controlled study. Archives of Physical Medicine and Rehabilitation, 94(1), 1-8.

Ziemann, U., Muellbacher, W., Hallett, M., \& Cohen, L. G. (2001). Modulation of practice-dependent plasticity in human motor cortex. Brain, 124(6), 1171-1181. 